\title{
LncRNA-mediated SIRT1/FoxO3a and SIRT1/p53 signaling pathways regulate type II alveolar epithelial cell senescence in patients with chronic obstructive pulmonary disease
}

\author{
CHAO GU ${ }^{1,2}$, YAQING LI ${ }^{1}$, JIALIANG LIU ${ }^{2}$, XIWANG YING ${ }^{1}$, YUANSHUN LIU ${ }^{1}$, \\ JIANPING YAN ${ }^{1}$, CHUN CHEN $^{1}$, HONGBIN ZHOU ${ }^{1}$, LIMING CAO $^{1}$ and YINGYU MA ${ }^{3}$ \\ ${ }^{1}$ Department of Respiratory Medicine, Zhejiang Provincial People's Hospital, Hangzhou, Zhejiang 310014; \\ ${ }^{2}$ Department of Respiratory Medicine, The First Hospital of Jiaxing, Jiaxing, Zhejiang 314000; \\ ${ }^{3}$ Clinical Research Institute, Zhejiang Provincial People's Hospital, Hangzhou, Zhejiang 310014, P.R. China
}

Received January 12, 2016; Accepted February 9, 2017

DOI: $10.3892 / \mathrm{mmr} .2017 .6367$

\begin{abstract}
The loss of alveolar structure and airspace enlargement are major pathological changes in chronic obstructive pulmonary disease (COPD). Type II alveolar epithelial cells (AECII) are involved in maintaining lung tissue repair and alveolar homeostasis. Long non-coding RNAs (lncRNAs) are involved in multi-regulating gene transcription, affecting processes including embryonic development, cell differentiation and cellular senescence. The primary aim of the present study was to explore the mechanisms of AECII senescence regulated by lncRNA-mediated sirtuin 1 (SIRT1) and forkhead box $\mathrm{O} 3 \mathrm{a}$ (FoxO3a) signaling pathways in patients with COPD. Lung tissues from patients with COPD exhibited pathological characteristics and significantly increased senescence-associated $\beta$-galactosidase activity. Furthermore, the expression levels of senescence-associated lncRNA1 (SAL-RNA1), SIRT1 and FoxO3a were reduced, but SAL-RNA2, SAL-RNA3, p53 and $\mathrm{p} 21$ were upregulated in the lung tissues of patients with COPD compared with control. The results of the present study indicated that lncRNA-mediated SIRT1/p53 and FoxO3a signaling pathways may regulate AECII senescence in the pathogenesis of COPD, which may provide a novel experimental basis for the treatment of COPD.
\end{abstract}

\section{Introduction}

Loss of elastic recoil, perpetual destruction of alveolar structure and airspace enlargement are the pathological characteristics of patients with chronic obstructive pulmonary disease

Correspondence to: Dr Yaqing Li, Department of Respiratory Medicine, Zhejiang Provincial People's Hospital, 158 Shangtang Road, Hangzhou, Zhejiang 310014, P.R. China

E-mail: lidoctor03@126.com

Key words: long non-coding RNA, sirtuin 1, cellular senescence, alveolar epithelial cells, chronic obstructive pulmonary disease
(COPD) (1). Senescence and apoptosis of alveolar epithelial cells results in the destruction of the alveolar structure, which may contribute to the pathogenesis of COPD (2-4). However, there is no effective treatment to prevent the destruction of the alveolar structure in patients with COPD. As progenitors of type I alveolar epithelial cells (AECI) in mammals, type II alveolar epithelial cells (AECII) are involved in synthesizing and secreting pulmonary surfactant proteins, maintaining alveolar homeostasis, reducing surface tension of the alveoli, improving lung tissue repair and gas exchange $(5,6)$. Although stem cells are involved in lung repair $(7,8)$, there still is no effective method to prevent AECII senescence and damage.

As a nicotinamide adenine dinucleotide (NAD) ${ }^{+}$-dependent class III histone deacetylase, sirtuin 1 (SIRT1) is an important member of the sirtuin protein family $(9,10)$. SIRT1 deacetylate $\mathrm{H} 1, \mathrm{H} 3$ and $\mathrm{H} 4$ histones, forkhead box O 3a (FoxO3a), p53 and nuclear factor- $\mathrm{\kappa B}$, which are involved in the regulation of signaling pathways of stress resistance, inflammation, cellular senescence, apoptosis and proliferation (11-13). FoxO3a is an important member of the forkhead box $\mathrm{O}$ subfamily and is a non-histone substrate of SIRT1. p53, another non-histone substrate of SIRT1, activates the transcription of p21 and inhibit the activity of cyclin-dependent kinases, which resulted in accelerating cellular senescence (14).

Long non-coding RNAs (lncRNAs) are non-protein-coding RNAs that are longer than 200 nucleotides (15), which are involved in regulating gene transcription and thus processes including embryonic development, cell differentiation and cellular senescence $(16,17)$. Previous studies have demonstrated that $\operatorname{lncRNAs}$ directly regulate cell functions, and are associated with multiple diseases (18). Abdelmohsen et al (19) indicated that the expression levels of senescence-associated lncRNA1 (SAL-RNA1) and SIRT1 were downregulated, but SAL-RNA2, SAL-RNA3, p53 and p21 were upregulated in senescent fetal lung-derived WI-38 human diploid fibroblasts (WI-38 HDFs). Furthermore, inhibiting SAL-RNA1 levels enhances the phenotypic characteristics of senescent WI-38 HDFs, which increases senescence-associated $\beta$-galactosidase (SA- $\beta$-gal) activity and 553 expression. 
However, it remains unknown whetherSAL-RNA-mediated SIRT1/p53 and the FoxO3a signaling pathways are involved in regulating AECII senescence in the pathogenesis of COPD. The present study investigated whether AECII senescence in lung tissues promoted the pathogenesis of COPD.

\section{Materials and methods}

Patients and specimens. A total of 34 patients who underwent lung surgery at Zhejiang Provincial People's Hospital (Hangzhou, China) from January 2014 to April 2015 were enrolled in the present study. These patients included 28 men and 6 women, ranging from 41-81 years old, with a median age of 66.5 years. None of the patients received preoperative chemotherapy, radiotherapy or intravenous corticosteroid therapy, and these patients did not exhibit failure of the heart, lung, brain or kidney, or any hematological diseases. Patients diagnosed with respiratory diseases other than COPD, including idiopathic pulmonary fibrosis and asthma, were excluded. According to the global strategy for the diagnosis of chronic obstructive pulmonary disease (1), these patients were sorted into 2 groups as follows: A control group (22 patients) and a COPD group (12 patients, exposed to cigarette smoke for $>20$ years). Lung function of each patient was examined by spirometry, which was performed by a trained technician according to the American Thoracic Society/European Respiratory Society guidelines (20). The lung tissues, taken from a distance of $5 \mathrm{~cm}$ from the negative margin of cancer tissues, were collected immediately following surgical resection and frozen instantly in liquid nitrogen or immersed in paraformaldehyde solution. The present study was approved by the Ethics Committee of Zhejiang Provincial People's Hospital (Hangzhou, China). Informed consent was obtained from all patients.

Histological examinations. Lungs were immersed in paraformaldehyde for $24 \mathrm{~h}$, then embedded in paraffin and cut into $4 \mu \mathrm{m}$ thick sections. A total of 3 discontinuous paraffin-embedded sections from each lung tissue sample were stained with hematoxylin and eosin (H\&E) in order to assess the morphological changes in the lungs. A total of 5 fields of view from each of the 3 sections from each lung sample were assessed using a light microscope (Olympus Corporation, Tokyo, Japan). The mean linear intercept (MLI), the mean alveolar airspace (MAA) and the number of alveolar counted per unit area (MAN) were obtained. MLI indicates the average distance between opposing walls of a single alveolus and is a measure of pulmonary airspace enlargement. MAA is also a measure of pulmonary airspace enlargement, while MAN is a measure of pulmonary airspace density.

Senescence-associated- $\beta$-galactosidase (SA- $\beta$-gal) activity assay. SA- $\beta$-gal activity was assessed using an in situ $\beta$-galactosidase staining kit (Beyotime Institute of Biotechnology, Shanghai, China) according to the manufacturer's protocol. Lung tissues were fixed in $\beta$-galactosidase stationary solution for $15 \mathrm{~min}$, then washed 3 times for $10 \mathrm{~min}$ each in PBS. Sections were then incubated with $1 \mathrm{ml}$ staining solution mixture (10 $\mu$ l staining solution A, $10 \mu$ l staining solution $\mathrm{B}, 930 \mu \mathrm{l}$ staining solution $\mathrm{C}$ and $50 \mu \mathrm{l} \mathrm{X}$-gal solution) for
$2 \mathrm{~h}$ at $37^{\circ} \mathrm{C}$. Following 3 washes with PBS, 5 fields of view from each of the 3 sections from each lung sample were examined using a light microscope (Olympus Corporation).

Reverse transcription-quantitative polymerase chain reaction $(R T-q P C R)$. Total RNA was extracted from each lung sample using TRIzol reagent (Invitrogen; Thermo Fisher Scientific, Inc., Waltham, MA, USA) according to the manufacturer's protocol. The final RNA purity and concentrations were determined using a spectrophotometer. cDNA was synthesized from the total RNA using the PrimeScript ${ }^{\mathrm{TM}}$ RT reagent kit with gDNA eraser (Takara Bio, Inc., Otsu, Japan) according to the manufacturer's protocol. qPCR analysis was performed using SYBR-Green qPCR kit (Takara Bio, Inc.) and the Agilent MX3000P qPCR system (Agilent Technologies, Inc., Santa Clara, CA, USA). The thermocycling conditions were as follows: Initial denaturation at $95^{\circ} \mathrm{C}$ for $15 \mathrm{~min}$, followed by 45 cycles of denaturation at $95^{\circ} \mathrm{C}$ for $15 \mathrm{sec}$, annealing at $58^{\circ} \mathrm{C}$ for $15 \mathrm{sec}$, and elongation at $72^{\circ} \mathrm{C}$ for $1 \mathrm{~min}$. The following primers were used (Invitrogen; Thermo Fisher Scientific, Inc.): SAL-RNA1, forward 5'-AGGCTGCCATCTCACCCT CATAC-3' and reverse 5'-TCCTTACCTCTTCCCTTCCAC CC-3'; SAL-RNA2, forward 5'-GGATGCTGTGAGCTTTGT GA-3' and reverse 5'-GAAACCCCCAGAGCTGAGAC-3'; SAL-RNA3, forward 5'-ACTGCTGGGATAACGGTGAC-3' and reverse 5'-TCTGTGCTCAGCTCTGCAAT-3'; SIRT1, forward 5'-GCAGATTAGTAGGCGGCTTG-3' and reverse 5'-ACTTTCATCCTCCATGGGTTC-3'; p53, forward 5'-ACC ACCATCCACTACAACTACAT-3' and reverse 5'-CAGGAC AGGCACAAACACG-3'; FOXO3a, forward 5'-TGGCAA GCACAGAGTTGGATGA-3' and reverse 5'-TGGCGGGAG CGTGATGTTAT-3'; p21, forward 5'-ACTTTGATTAGCAGC GGAACA-3' and reverse 5'-GAAAACAGTCCAGGCCAG TATG-3'; and glyceraldehyde 3-phosphate dehydrogenase (GAPDH), forward 5'-TGAAGGTCGGAGTCAACGG-3' and reverse 5'-CTGGAAGATGGTGATGGGATT-3'. All analyses were performed in triplicate. GADPH was used as a reference gene. The results were quantified using the $2^{-\Delta \Delta C q}$ method (21).

Western blotting. Proteins were extracted from each lung sample by homogenizing the samples in ice-cold lysis buffer [50 mmol/1 Tris (pH 7.4), $150 \mathrm{mmol} / 1 \mathrm{NaCl}, 0.1 \%$ sodium dodecyl sulfate, $1 \mathrm{mmol} / 1$ EDTA, $1 \%$ sodium deoxycholate, and $1 \%$ Triton X-100; Invitrogen; Thermo Fisher Scientific, Inc.] containing a protease inhibitor cocktail $(1 \mathrm{mmol} / \mathrm{l}$ phenylmethylsulfonyl fluoride, $1 \mathrm{mg} / \mathrm{l}$ leupeptin and $1 \mathrm{mg} / \mathrm{l}$ aprotinin; Beyotime Institute of Biotechnology). The protein concentration in the samples was determined using a micro bicinchoninic acid protein assay kit (Pierce; Thermo Fisher Scientific, Inc.) according to the manufacturer's protocol. Next, equal amounts of protein $(20 \mu \mathrm{g})$ from each sample were heated at $100^{\circ} \mathrm{C}$ for 5 min and then separated by electrophoresis on $8 \%$ sodium dodecyl sulfate-polyacrylamide gels. The separated proteins were electrotransferred to nitrocellulose membranes and blocked with Tris-buffered saline containing 0.1\% Tween-20 (TBS-T) and 5\% bovine serum albumin (BSA; Sigma-Aldrich; Merck KGaA, Darmstadt, Germany) for $2 \mathrm{~h}$ at room temperature. The membranes were then incubated with primary antibodies against p53 (\#9282; 1:1,000 in TBS-T; Cell Signaling Technology, Inc., Danvers, MA, USA), p21 (sc-397; 1:500 in TBS-T; Santa Cruz 
Biotechnology, Inc., Dallas, TX, USA), FoxO3a (\#12829; 1:1,000 in TBS-T; Cell Signaling Technology, Inc.) SIRT1 (\#2493; 1:1,000 in TBS-T; Cell Signaling Technology) and $\beta$-actin (ab8227; 1:1,000; Abcam, Cambridge, UK) overnight at $4^{\circ} \mathrm{C}$ on an orbital shaker. Following washing 3 times for 10 min each in TBS-T, the membranes were incubated with horseradish peroxidase-conjugated goat anti-rabbit immunoglobulin G (\#35552; 1:5,000 in TBS-T; Pierce; Thermo Fisher Scientific, Inc.) for $1 \mathrm{~h}$ at room temperature on an orbital shaker. Following 5 washes with TBS-T, immunoreactive bands on the membrane were detected using an enhanced chemiluminescence solution (GE Healthcare Life Sciences, Chalfont, UK), visualized by means of X-ray-film exposure, and analyzed using an UVP-GDS8000 gel-analysis system (Ultra-Violet Products, Ltd., Cambridge, UK). Protein expression levels were analyzed by densitometry and the values were normalized relative to those measured for $\beta$-actin, which was used as the loading control.

SIRT1 deacetylase activity assay. SIRT1 deacetylase activity was measured using the SIRT1 fluorimetric activity assay kit (Enzo Life Sciences, Inc., Farmingdale, NY, USA) according to the manufacturer's protocol. SIRT1 was immunoprecipitated as described previously (22). Following washing with PBS, Fluor de Lys substrate and $\mathrm{NAD}^{+}$were added to the SIRT1-conjugated beads and incubated for $90 \mathrm{~min}$ at $37^{\circ} \mathrm{C}$. Then, the substrate-SIRT1 mixture was placed on a white 96-well plate, and the Fluor de Lys developer II reagent was added to the 96 wells for $30 \mathrm{~min}$ at $37^{\circ} \mathrm{C}$. Finally, the plate was analyzed by a microplate reading fluorimeter (M200; Tecan Group, Ltd., Männedorf, Switzerland) at $405 \mathrm{~nm}$.

Statistical analyses. Statistical analyses were performed using SPSS 17.0 software (SPSS Inc., Chicago, IL, USA). Data were expressed as the mean \pm standard deviation. The significance of difference between groups was determined by means of independent-sample Student's t-tests. $\mathrm{P}<0.05$ was considered to indicate a statistically significant difference.

\section{Results}

Lung function examination. The lung function of each patient was examined by spirometry. There were no significant differences between patients from the control group and the COPD group in forced vital capacity (FVC; P>0.05; Table I). However, the ratio of forced expiratory ventilation in $1 \mathrm{sec}$ to $\mathrm{FVC}$ $\left(\mathrm{FEV}_{1} / \mathrm{FVC}\right)$ and $\% \mathrm{FEV}_{1}$ in patients with COPD was significantly reduced compared with the control group $(\mathrm{P}<0.001$; Table I).

Lung histological characteristics. The histological characteristics of lung tissues from the control group and the COPD group were analyzed by H\&E staining. Compared with the control group (Fig. 1A), the airspace was visibly enlarged and the number of alveoli was decreased in lung tissues of the COPD group (Fig. 1B). The lung samples from the COPD group also exhibited merged alveoli and the formation of bullae, which was in accord with the pathological characteristics of COPD (Fig. 1B). Quantitative analyses of lung histomorphology revealed that the MLI and MAA of the COPD group was significantly higher compared with the control group $(\mathrm{P}<0.05$;
Table I. Lung function examination in patients with COPD.

\begin{tabular}{lccr}
\hline Variable & Control & COPD & P-value \\
\hline FVC (L) & $2.47 \pm 0.59$ & $2.44 \pm 0.63$ & 0.789 \\
FEV $_{1} /$ FVC $(\%)$ & $82.39 \pm 5.59$ & $64.13 \pm 6.83$ & $<0.001$ \\
FEV $_{1}, \%$ predicted & $92.15 \pm 10.11$ & $63.28 \pm 14.09$ & $<0.001$ \\
\hline
\end{tabular}

COPD, chronic obstructive pulmonary disease; FVC, forced vital capacity; $\mathrm{FEV}_{1}$, forced expiratory ventilation in $1 \mathrm{sec}$. Data are presented as the mean \pm standard deviation.

Table II. Quantitative analyses of lung histomorphology in patients with COPD.

\begin{tabular}{lccc}
\hline Group & MLI $(\mu \mathrm{M})$ & MAA $\left(\mu \mathrm{m}^{2}\right)$ & MAN $\left(\mathrm{mm}^{2}\right)$ \\
\hline Control & $35.26 \pm 2.47$ & $3166.57 \pm 127.32$ & $264.76 \pm 25.81$ \\
COPD & $189.16 \pm 15.22^{\mathrm{a}}$ & $20681.16 \pm 768.86^{\mathrm{a}}$ & $50.44 \pm 4.85^{\mathrm{a}}$ \\
\hline
\end{tabular}

COPD, chronic obstructive pulmonary disease; MLI, mean linear intercept; MAA, mean alveolar airspace; MAN, the number of alveolar counted per unit area. Data are presented as the mean \pm standard deviation. ${ }^{\mathrm{a}} \mathrm{P}<0.05$ vs. control group.

Table II) and the MAN was significantly decreased in the COPD group compared with the control group $(\mathrm{P}<0.05$; Table II).

SA- $\beta$-gal activity. SA- $\beta$-gal activity was assayed in lung samples to analyze whether cellular senescence was exhibited in lung tissues of the patients with COPD. The cells in lung tissues with blue color were considered SA- $\beta$-gal positive, and so senescent. Compared with the control group (Fig. 2A), SA- $\beta$-gal activity was visibly increased in the COPD group (Fig. 2B) Most SA- $\beta$-gal-positive cells were located at the edges of alveoli (Fig. 2B).

LncRNA expression of SAL-RNAs. The IncRNA expression of SAL-RNA1, SAL-RNA2 and SAL-RNA3 was measured by RT-qPCR. SAL-RNA2 and SAL-RNA3 mRNA expression levels were significantly increased in the lung tissues of the COPD group compared with the control group $(\mathrm{P}<0.05$; Fig. 3$)$. However, the SAL-RNA1 level in the COPD group was significantly decreased compared with the control group $(\mathrm{P}<0.05$; Fig. 3).

mRNA expression of SIRT1, FoxO3a, p53 and p21. p53 and p21 mRNA expression levels were significantly increased in lung tissues in the patients with COPD compared with the control group $(\mathrm{P}<0.05$; Fig. 4). By contrast, SIRT1 and FoxO3a mRNA expression levels in the COPD group were significantly decreased compared with the control group ( $\mathrm{P}<0.05$; Fig. 4).

Protein expression and SIRT1 deacetylase activity. SIRT1, FoxO3a, p53 and p21 protein expression levels were visualized by western blotting. SIRT1 and FoxO3a protein levels were 

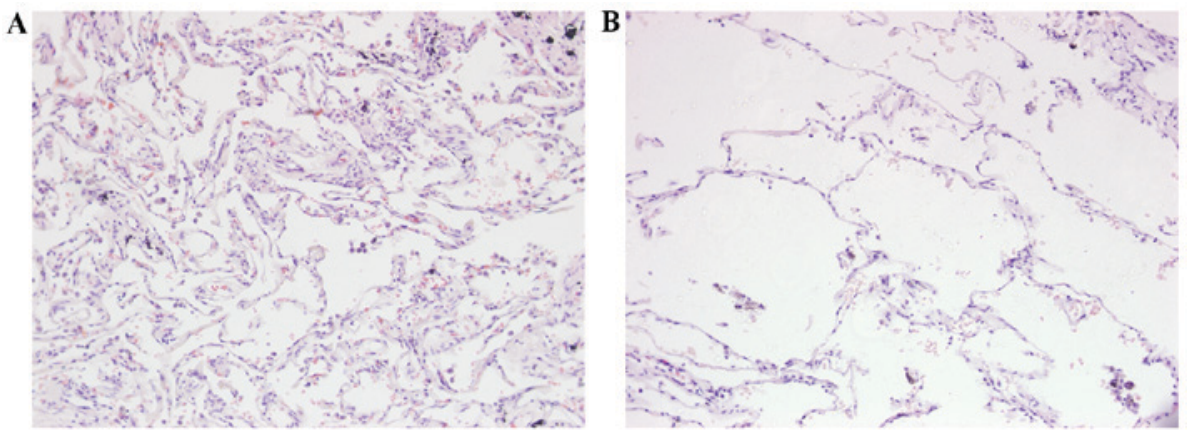

Figure 1. Lung histological characteristics of (A) the control group and (B) the COPD group. The airspace was visibly enlarged and the number of alveoli was decreased in the COPD group. Original magnification, x200. COPD, chronic obstructive pulmonary disease.
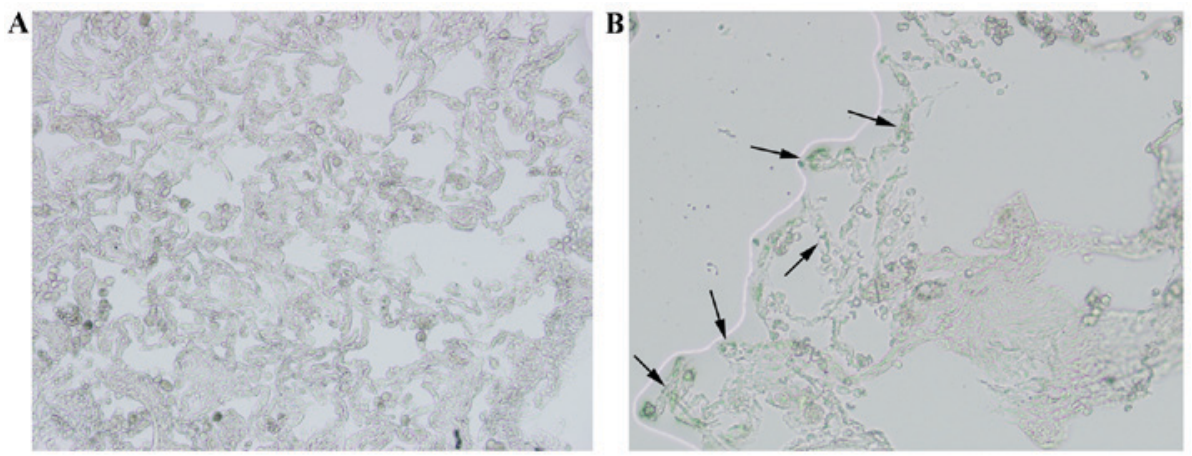

Figure 2. SA- $\beta$-gal activity of lung tissues in (A) the control group and (B) the COPD group. SA- $\beta$-gal activity was visibly increased in COPD group, and most SA- $\beta$-gal-positive cells were located in the corner of the alveoli. The arrows indicate SA- $\beta$-gal-positive cells. Original magnification, $x 200$. SA- $\beta$-gal, senescence associated- $\beta$-galactosidase; COPD, chronic obstructive pulmonary disease.

visibly reduced in the COPD group compared with the control group, while p53 and p21 protein levels were visibly upregulated in the COPD group compared with the control group (Fig. 5A). Accompanying the downregulation in SIRT1 protein levels, there was a significant reduction of SIRT1 activity in the COPD group compared with the control group $(\mathrm{P}<0.05 ;$ Fig. $5 \mathrm{~B})$.

\section{Discussion}

COPD is the fourth leading cause of death globally (1). Cigarette smoking is a major risk factor in the development of COPD. AECII are involved in the maintenance and repair of lung tissue and alveolar homeostasis $(5,6)$. Cellular senescence and apoptosis of alveolar epithelial cells in lung tissues are important characteristics of COPD pathogenesis $(2,23,24)$. SA- $\beta$-gal is considered an important biomarker for cellular senescence (25). In the present study, the airspace was visibly enlarged and the number of alveoli decreased in the lung tissues of patients with COPD. The results also revealed that the MLI, MAA and SA- $\beta$-gal activity were significantly increased and MAN was significantly decreased in the COPD group compared to the control group. Cigarette smoke exposure induces cellular senescence and apoptosis of alveolar cells, and results in air space enlargement $(23,26)$.

Previous studies have demonstrated that the expression of SIRT1 is significantly decreased in the lung tissues of COPD patients as well as in lung samples of rats with emphysema (27-29). SIRT1 is associated with cellular senescence in the development of emphysema, and the activation of SIRT1 may be a target for COPD/emphysema treatment (30). Activation of SIRT1 increases SIRT1 expression, which is associated with the upregulation of FoxO3, downregulation of p53 and inhibition of the AECII apoptosis (29). Previous studies have demonstrated that SIRT1 is an antiaging protein, and is reduced in the lung tissues of patients with COPD $(27,28)$. Cellular senescence mediated by SIRT1 participates in the progression of COPD, but the positive effects of SIRT1 on AECII senescence in patients with COPD remain elusive.

p53 is a tumor suppressor protein, that possesses the ability to induce cellular senescence by activating p 21 expression. SIRT1 possesses the biochemical potential to deacetylate lysine residues of $\mathrm{p} 53$ or regulate the gene promoter of $\mathrm{p} 53$, which inhibits p53 activity and reduces cellular senescence (31). FoxO3a is an important member of the forkhead box O subfamily, which is a non-histone substrate of SIRT1. Protein complexes with the combination of SIRT1 and FoxO3a may regulate apoptosis and cellular senescence (32). SIRT1/FoxO3a and SIRT1/p53 pathways may regulate the cell cycle inhibitors (p16 and p21) to accelerate cellular senescence (33).

LncRNAs are associated with multiple diseases, including Alzheimer's disease (34), coronary artery disease (35), prostate cancer (36) and lung cancer (37), but few IncRNA are associated with COPD. A previous study (19) revealed that high levels of SAL-RNA1 are expressed in early-passage WI-38 HDFs, but the expression levels of SAL-RNA2 and SAL-RNA3 are low. Following 52 population doublings SAL-RNA1 and SIRT1 expression levels are downregulated, but SAL-RNA2, SAL-RNA3, p53 and p21 expression levels are upregulated in 


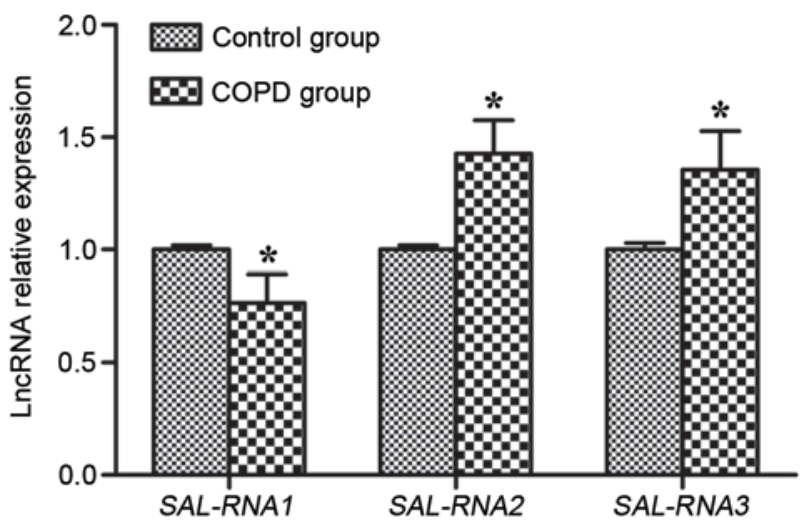

Figure 3. LncRNA SAL-RNA1, SAL-RNA2 and SAL-RNA3 expression in lung tissues, measured by reverse transcription-quantitative polymerase chain reaction and quantified with the $2^{-\Delta \Delta C q}$ method. All analyses were performed in triplicate. Values are presented as the mean + standard deviation. "P $<0.05$ vs. control group. LncRNA, long non-coding RNA; SAL-RNA, senescence-associated long non-coding RNA; COPD, chronic obstructive pulmonary disease.

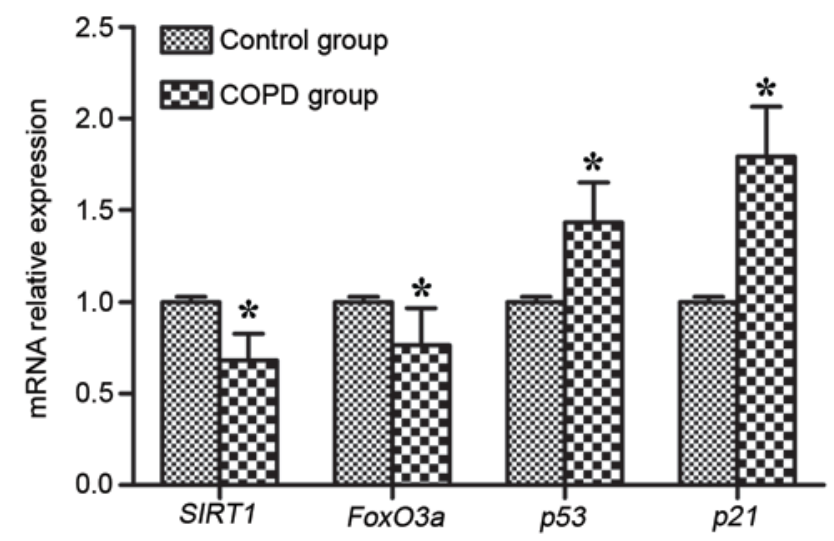

Figure 4. mRNA expression in lung tissues. SIRT1, FoxO3a, p53 and p21 mRNA expression levels were analyzed by reverse transcription-quantitative polymerase chain reaction and quantified with the $2^{-\Delta \Delta C q}$ method. All analyses were performed in triplicate. Values are presented as the mean + standard deviation. " $\mathrm{P}<0.05$ vs. control group. SIRT1, sirtuin 1; FoxO3a, forkhead box $\mathrm{O} 3 \mathrm{a} ; \mathrm{COPD}$, chronic obstructive pulmonary disease.

the senescent WI-38 HDFs. Furthermore, inhibiting SAL-RNA1 levels may enhance the phenotypic characteristics of senescent WI-38 HDFs, which may increase SA- $\beta$-gal activity and p53 expression. In the present study, SAL-RNA1, SIRT1 and FoxO3a expression was downregulated, but SAL-RNA2, SAL-RNA3, p53 and p21 expression was upregulated in lung tissues of the COPD group compared with the control group. Thus, the results of the present study indicated that the SIRT1/FoxO3a and SIRT1/p53 pathways were mediated by SAL-RNA1, SAL-RNA2 and SAL-RNA3, which may regulate AECII senescence in the pathogenesis of COPD, and may provide novel targets for COPD therapy.

\section{Acknowledgements}

The present study was supported by grants from the National Natural Science Foundation of China (grant nos. 81470241, 81000016 and 81470109) and the Foundation of Science
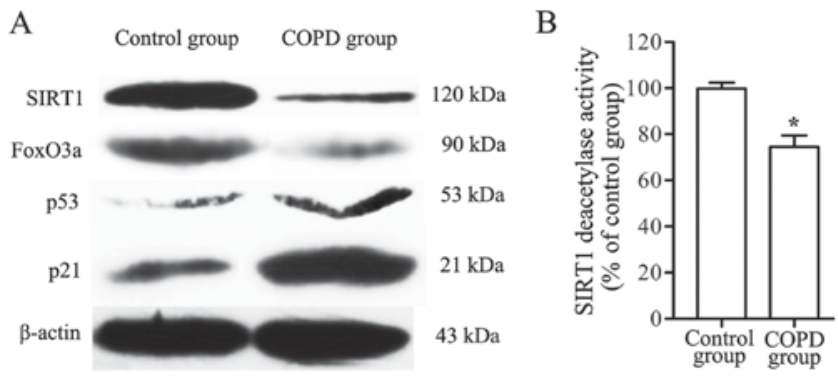

Figure 5. (A) SIRT1, FoxO3a, p53 and p21 protein levels in lung tissue samples, analyzed by western blot, with $\beta$-actin as a loading control. (B) SIRT1 deacetylase activity in lung samples. All analyses were performed in triplicate. Values are presented as the mean + standard deviation. ${ }^{*} \mathrm{P}<0.05$ vs. control group. SIRT1, sirtuin 1; FoxO3a, forkhead box O 3a; COPD, chronic obstructive pulmonary disease.

and Technology Department of Zhejiang Province (grant no. 2014C37022).

\section{References}

1. Global Initiative for Chronic Obstructive Lung Disease: Global strategy for the diagnosis, management and prevention of chronic obstructive pulmonary disease. Seattle: GOLD, Available from URL: http://www.goldcopd.com/, 2015.

2. Tsuji T, Aoshiba K and Nagai A: Alveolar cell senescence exacerbates pulmonary inflammation in patients with chronic obstructive pulmonary disease. Respiration 80: 59-70, 2010.

3. Siganaki M, Koutsopoulos AV, Neofytou E, Vlachaki E, Psarrou M, Soulitzis N, Pentilas N, Schiza S, Siafakas NM and Tzortzaki EG: Deregulation of apoptosis mediators' p53 and bcl 2 in lung tissue of COPD patients. Respir Res 11: 46, 2010.

4. Zhao CZ, Fang XC, Wang D, Tang FD and Wang XD: Involvement of type II pneumocytes in the pathogenesis of chronic obstructive pulmonary disease. Respir Med 104: 1391-1395, 2010.

5. Whitsett JA, Wert SE and Weaver TE: Alveolar surfactant homeostasis and the pathogenesis of pulmonary disease. Annu Rev Med 61: 105-119, 2010.

6. Hoffman AM and Ingenito EP: Alveolar epithelial stem and progenitor cells: Emerging evidence for their role in lung regeneration. Curr Med Chem 19: 6003-6008, 2012.

7. Li Y, Xu W, Yan J, Xia Y, Gu C, Ma Y and Tao H: Differentiation of human amniotic fluid-derived mesenchymal stem cells into type II alveolar epithelial cells in vitro. Int J Mol Med 33: 1507-1513, 2014.

8. Li Y, Gu C, Xu W, Yan J, Xia Y, Ma Y, Chen C, He X and Tao H: Therapeutic effects of amniotic fluid-derived mesenchymal stromal cells on lung injury in rats with emphysema. Respir Res 15: 120, 2014.

9. Milne JC and Denu JM: The sirtuin family: Therapeutic targets to treat diseases of aging. Curr Opin Chem Biol 12: 11-17, 2008.

10. Bordo D: Structure and evolution of human sirtuins. Curr Drug Targets 14: 662-665, 2013.

11. Lavu S, Boss O, Elliott PJ and Lambert PD: Sirtuins--novel therapeutic targets to treat age-associated diseases. Nat Rev Drug Discov 7: 841-853, 2008.

12. Finkel T, Deng CX and Mostoslavsky R: Recent progress in the biology and physiology of sirtuins. Nature 460: 587-591, 2009.

13. Satoh A, Stein L and Imai S: The role of mammalian sirtuins in the regulation of metabolism, aging and longevity. Handb Exp Pharmacol 206: 125-162, 2011.

14. Guterres FA, Martinez GR, Rocha ME and Winnischofer SM: Simvastatin rises reactive oxygen species levels and induces senescence in human melanoma cells by activation of $\mathrm{p} 53 / \mathrm{p} 21$ pathway. Exp Cell Res 319: 2977-2988, 2013.

15. Kung JT, Colognori D and Lee JT: Long noncoding RNAs: Past, present and future. Genetics 193: 651-669, 2013.

16. Batista PJ and Chang HY: Long noncoding RNAs: Cellular address codes in development and disease. Cell 152: 1298-1307, 2013. 
17. Ohsawa R, Seol JH and Tyler JK: At the intersection of non-coding transcription, DNA repair, chromatin structure and cellular senescence. Front Genet 4: 136, 2013.

18. Mercer TR, Dinger ME and Mattick JS: Long non-coding RNAs: Insights into functions. Nat Rev Genet 10: 155-159, 2009.

19. Abdelmohsen K, Panda A, Kang MJ, Xu J, Selimyan R, Yoon JH, Martindale JL, De S, Wood WH III, Becker KG and Gorospe M: Senescence-associated lncRNAs: Senescence-associated long noncoding RNAs. Aging Cell 12: 890-900, 2013.

20. Miller MR, Hankinson J, Brusasco V, Burgos F, Casaburi R, Coates A, Crapo R, Enright P, van der Grinten CP, Gustafsson P, et al: Standardisation of spirometry. Eur Respir J 26: 319-338, 2005.

21. Livak KJ and Schmittgen TD: Analysis of relative gene expression data using real-time quantitative PCR and the 2(-Delta Delta C(T)) Method. Methods 25: 402-408, 2001.

22. Caito S, Rajendrasozhan S, Cook S, Chung S, Yao H, Friedman AE, Brookes PS and Rahman I: SIRT1 is a redox-sensitive deacetylase that is post-translationally modified by oxidants and carbonyl stress. FASEB J 24: 3145-3159, 2010.

23. Farkas L, Farkas D, Warburton D, Gauldie J, Shi W, Stampfli MR, Voelkel NF and Kolb M: Cigarette smoke exposure aggravates air space enlargement and alveolar cell apoptosis in Smad3 knockout mice. Am J Physiol Lung Cell Mol Physiol 301: L391-L401, 2011.

24. Mimae T, Hagiyama M, Inoue $T$, Yoneshige A, Kato $T$, Okada M, Murakami Y and Ito A: Increased ectodomain shedding of lung epithelial cell adhesion molecule 1 as a cause of increased alveolar cell apoptosis in emphysema. Thorax 69: 223-231, 2014.

25. Gary RK and Kindell SM: Quantitative assay of senescence-associated beta-galactosidase activity in mammalian cell extracts. Anal Biochem 343: 329-334, 2005.

26. Hara H, Araya J, Takasaka N, Fujii S, Kojima J, Yumino Y, Shimizu K, Ishikawa T, Numata T, Kawaishi M, et al: Involvement of creatine kinase B in cigarette smoke-induced bronchial epithelial cell senescence. Am J Respir Cell Mol Biol 46: 306-312, 2012.

27. Rajendrasozhan S, Yang SR, Kinnula VL and Rahman I: SIRT1, an antiinflammatory and antiaging protein, is decreased in lungs of patients with chronic obstructive pulmonary disease. Am J Respir Crit Care Med 177: 861-870, 2008
28. Nakamaru Y, Vuppusetty C, Wada H, Milne JC, Ito M, Rossios C, Elliot M, Hogg J, Kharitonov S, Goto H, et al: A protein deacetylase SIRT1 is a negative regulator of metalloproteinase-9. FASEB J 23: 2810-2819, 2009 .

29. Gu C, Li Y, Xu WL, Yan JP, Xia YJ, Ma YY, Chen C, Wang HJ and Tao HQ: Sirtuin 1 activator SRT1720 protects against lung injury via reduction of type II alveolar epithelial cells apoptosis in emphysema. COPD 12: 444-452, 2015.

30. Yao H, Chung S, Hwang JW, Rajendrasozhan S, Sundar IK, Dean DA, McBurney MW, Guarente L, Gu W, Rönty M, et al: SIRT1 protects against emphysema via FOXO3-mediated reduction of premature senescence in mice. J Clin Invest 122: 2032-2045, 2012.

31. Arunachalam G, Samuel SM, Marei I, Ding H and Triggle CR: Metformin modulates hyperglycaemia-induced endothelial senescence and apoptosis through SIRT1. Br J Pharmacol 171: 523-535, 2014.

32. Ganesan S, Unger BL, Comstock AT, Angel KA, Mancuso P, Martinez FJ and Sajjan US: Aberrantly activated EGFR contributes to enhanced IL-8 expression in COPD airways epithelial cells via regulation of nuclear FoxO3A. Thorax 68: 131-141, 2013.

33. Furukawa A, Tada-Oikawa S, Kawanishi S and Oikawa S: H2O2 accelerates cellular senescence by accumulation of acetylated p53 via decrease in the function of SIRT1 by NAD ${ }^{+}$depletion. Cell Physiol Biochem 20: 45-54, 2007.

34. Faghihi MA, Modarresi F, Khalil AM, Wood DE, Sahagan BG, Morgan TE, Finch CE, St Laurent G III, Kenny PJ and Wahlestedt C: Expression of a noncoding RNA is elevated in Alzheimer's disease and drives rapid feed-forward regulation of beta-secretase. Nat Med 14: 723-730, 2008.

35. Visel A, Zhu Y, May D, Afzal V, Gong E, Attanasio C, Blow MJ, Cohen JC, Rubin EM and Pennacchio LA: Targeted deletion of the $9 \mathrm{p} 21$ non-coding coronary artery disease risk interval in mice. Nature 464: 409-412, 2010.

36. Chung S, Nakagawa H, Uemura M, Piao L, Ashikawa K, Hosono N, Takata R, Akamatsu S, Kawaguchi T, Morizono T, et al: Association of a novel long non-coding RNA in 8q24 with prostate cancer susceptibility. Cancer Sci 102: 245-252, 2011.

37. Schmidt LH, Spieker T, Koschmieder S, Schäffers S, Humberg J, Jungen D, Bulk E, Hascher A, Wittmer D, Marra A, et al: The long noncoding MALAT-1 RNA indicates a poor prognosis in non-small cell lung cancer and induces migration and tumor growth. J Thorac Oncol 6: 1984-1992, 2011. 